\title{
Complete Response with Incomplete Bone Marrow Recovery
}

National Cancer Institute

\section{Source}

National Cancer Institute. Complete Response with Incomplete Bone Marrow Recovery. NCl Thesaurus. Code C103385.

The disappearance of all signs of cancer in response to treatment accompanied by incomplete bone marrow recovery, as evidenced by persistent anemia, thrombocytopenia or neutropenia. 